\title{
A Landmark-Based Brain Conformal Parametrization with Automatic Landmark Tracking Technique
}

\author{
Lok Ming Lui ${ }^{1}$, Yalin Wang ${ }^{1,2}$, Tony F. Chan ${ }^{1}$, and Paul M. Thompson ${ }^{2}$ \\ ${ }^{1}$ Department of Mathematics, UCLA \\ ${ }^{2}$ Laboratory of Neuroimaging, Department of Neurology, UCLA \\ \{malmlui, ylwang, chan\}@math.ucla.edu, \\ thompson@loni.ucla.edu
}

\begin{abstract}
In this paper, we present algorithms to automatically detect and match landmark curves on cortical surfaces to get an optimized brain conformal parametrization. First, we propose an automatic landmark curve tracing method based on the principal directions of the local Weingarten matrix. Our algorithm obtains a hypothesized landmark curves using the Chan-Vese segmentation method, which solves a Partial Differential Equation (PDE) on a manifold with global conformal parameterization. Based on the global conformal parametrization of a cortical surface, our method adjusts the landmark curves iteratively on the spherical or rectangular parameter domain of the cortical surface along its principal direction field, using umbilic points of the surface as anchors. The landmark curves can then be mapped back onto the cortical surface. Experimental results show that the landmark curves detected by our algorithm closely resemble these manually labeled curves. Next, we applied these automatically labeled landmark curves to generate an optimized conformal parametrization of the cortical surface, in the sense that homologous features across subjects are caused to lie at the same parameter locations in a conformal grid. Experimental results show that our method can effectively help in automatically matching cortical surfaces across subjects.
\end{abstract}

\section{Introduction}

Parametrization of the cortical surfaces is an important process in the brain mapping research for data comparison. In this paper, we propose an automatic way to parametrize the cortical surface that matches important anatomical features. Important anatomical features on the cortical surface are usually represented by landmark curves, called sulcal/gyral curves. It is extremely time-consuming to label these landmark curves manually, especially when large dataset must be analyzed. We therefore propose an algorithm to detect these feature curves automatically. Given a global conformal parametrization of the cortical surface, we fix two endpoints, called the anchor points, based on umbilic points of the curvature field. We then trace the landmark curve by iteratively adjusting its path in the spherical or rectangular parameter domain of the cortex, along one of the two 
principal direction fields. Using the parameterization, the landmark curves can be mapped back onto the cortical surface in 3D. To speed up the iterative scheme, we propose a method to obtain a good initialization by extracting high curvature regions on the cortical surface using the Chan-Vese segmentation method [1]. This involves solving a PDE (Euler-Lagrange equation) on the cortical manifold using the global conformal parametrization. Finally, we use these automatically labelled landmark curves to create an optimized brain conformal mapping, which can match important anatomical features across subjects. This is based on the minimization of a combined energy functional $E_{\text {new }}=E_{\text {harmonic }}+\lambda E_{\text {landmark }}$.

\section{Previous Work}

Automatic detection of sulci landmark curves on the brain has been widely studied by different research groups. Prince et al. [2] proposed a method for automated segmentation of major cortical sulci on the outer brain boundary. This is based on a statistical shape model, which includes a network of deformable curves on the unit sphere, seeks geometric features such as high curvature regions, and labels such features via a deformation process that is confined within a spherical map of the outer brain boundary. Lohmann et al. 3. proposed an algorithm that can automatically detect and attribute neuroanatomical names to the cortical folds using image analysis methods applied to magnetic resonance data of human brains. The sulci basins are segmented using a region growing approach. Zeng et al. 4] proposed a method to automatic intrasulcal ribbon finding, by using the cortex segmentation with coupled surfaces via a level set method, where the outer cortical surface is embedded as the zero level set of a high-dimensional distance function.

Optimization of surface diffeomorphisms by landmark matching has been studied intensively. Gu et al. 5] proposed to optimize the conformal parametrization by composing an optimal Möbius transformation so that it minimizes the landmark mismatch energy. The resulting parameterization remains conformal. Joan et al. 6] proposed to generate large deformation diffeomorphisms of the sphere onto itself, given the displacements of a finite set of template landmarks. The diffeomorphism obtained can match the geometric features significantly but it is, in general, not a conformal mapping. Leow et al. 7] proposed a level set based approach for matching different types of features, including points and 2D or 3D curves represented as implicit functions. Cortical surfaces were flattened to the unit square. Nine sulcal curves were chosen and represented by the intersection of two level set functions They were used to constrain the warp of one cortical surface onto another. The resulting transformation was interpolated using a large deformation momentum formulation in the cortical parameter space, generalizing an elastic approach for cortical matching developed in Thompson et al. [8].

\section{Basic Mathematical Theory}

Firstly, a diffeomorphism $f: M \rightarrow N$ is a conformal mapping if it preserves the first fundamental form up to a scaling factor (the conformal factor). 
Mathematically, this means that $d s_{M}^{2}=\lambda f^{*}\left(d s_{N}^{2}\right)$, where $d s_{M}^{2}$ and $d s_{N}^{2}$ are the first fundamental form on surfaces $M$ and $N$, respectively and $\lambda$ is the conformal factor 9 .

Next, the normal curvature $\kappa_{n}$ of a Riemann surface in a given direction is the reciprocal of the radius of the circle that best approximates a normal slice of the surface in that direction, which varies in different directions. It follows that: $\kappa_{n}=\mathbf{v}^{T} \mathbb{I} \mathbf{v}=\mathbf{v}^{T}\left(\begin{array}{ll}e & f \\ f & g\end{array}\right) \mathbf{v}$ for any tangent vector $\mathbf{v}$. III is called the Weingarten matrix and is symmetric. Its eigenvalues and eigenvectors are called principal curvatures and principal directions respectively. The sum of the eigenvalues is the mean curvature. A point on the Riemann surface at which the Weingarten matrix has the same eigenvalues is called an umbilic point [10].
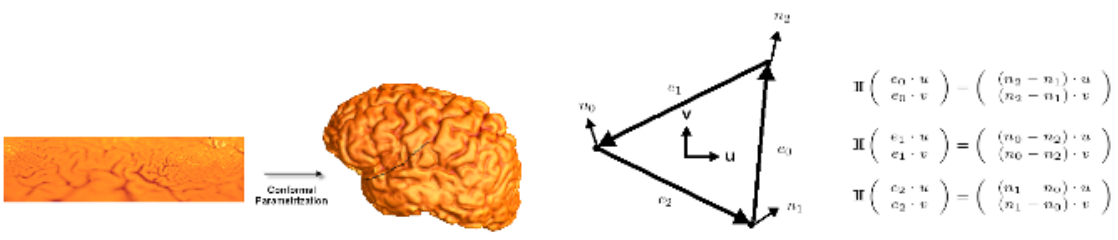

Fig. 1. Left: Conformal parametrization of the cortical surface onto the 2D rectangle. Right: A single face (triangle) of the triangulated mesh.

\section{Computation of Conformal Parameterization}

It is well known that any genus zero Riemann surfaces can be mapped conformally to a sphere. For the diffeomorphism between two genus zero surfaces, we can get a conformal map by minimizing the harmonic energy [5]. For high genus surfaces, $\mathrm{Gu}$ et al. 11] proposed an efficient approach to parameterize surfaces conformally using a set of connected $2 \mathrm{D}$ rectangles. They compute a holomorphic 1-form on the Riemann surface, using concepts from homology and cohomology group theory, and Hodge theory. (See Figure 1 left , [12])

\section{$5 \quad$ Algorithm for Automatic Landmark Tracking}

In this section, we discuss our algorithm for automatic landmark tracking.

\subsection{Computation of Principal Direction Fields from the Global Conformal Parametrization}

Denote the cortical surface by C. Let $\phi: D \rightarrow C$ be the global conformal parametrization of $C$ where $\mathrm{D}$ is a rectangular parameter domain. Let $\lambda$ be the conformal factor of $\phi$. Similar to Rusinkiewicz's work [13, we can compute the principal directions, and represent them on the parameter domain $D$. This is based on the following three steps: 


\section{Step 1: Per - Face Curvature Computation}

Let $u=\left(\begin{array}{c}\frac{1}{\sqrt{\lambda}} \\ 0\end{array}\right)$ and $v=\left(\begin{array}{c}0 \\ \frac{1}{\sqrt{\lambda}}\end{array}\right)$ be the directions of an orthonormal coordinate system for the tangent plane (represented in the parameter domain D). We can approximate the Weingarten matrix $\mathbb{I I}$ for each face (triangle). For a triangle with three well-defined directions (edges) together with the differences in normals in those directions (Refer to Figure 1 right), we have a set of linear constraints on the elements of the Weingarten matrix, which can be determined using the least square method.

\section{Step 2 : Coordinate system Transformation}

After we compute the Weingarten matrix on each face in the $\left(u_{f}, v_{f}\right)$ coordinate system, we can average it with contributions from adjacent triangles. This can be done by transforming the Weingarten matrix tensor into the vertex coordinate frame.

\section{Step3 : Weighting}

For each face $f$ which is adjacent to the vertex $p$, we take the weighting $w_{f, p}$ to be the area of $f$ divided by the squares of the lengths of the two edges that touch the vertex $p$.

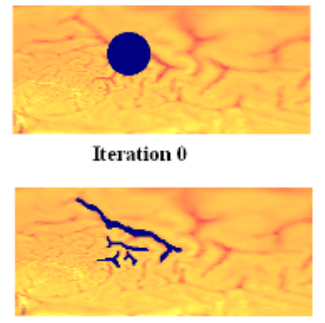

Final iteration
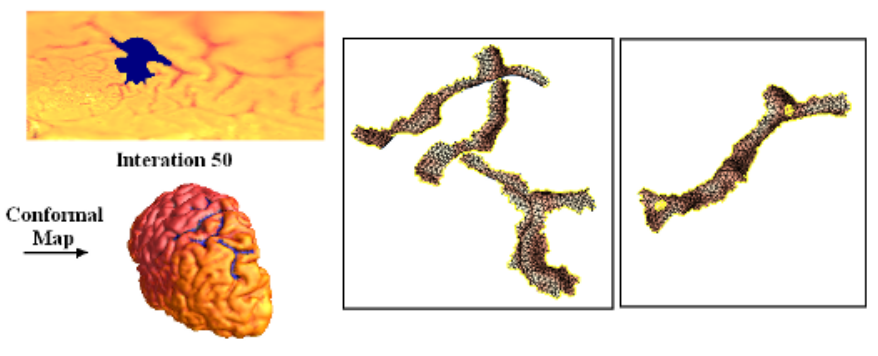

Fig. 2. Left :Sulcal curve extraction on the cortical surface by Chan-Vese segmentation. Right : Umbilic points are located on each sulci region, which are chosen as the end points of the landmark curves.

\subsection{Variational Method for Landmark Tracking}

Given the principal direction field $\vec{V}(t)$ with smaller eigenvalues on the cortical surface $C$, we propose a variational method to trace the sulcal landmark curve iteratively, after fixing two anchor points $(a \& b)$ on the sulci. Let $\phi: D \rightarrow C$ be the conformal parametrization of $\mathrm{C},\langle\cdot, \cdot\rangle$ to be its Riemannian metric and $\lambda$ to be its conformal factor. We propose to locate a curve $\vec{c}:[0,1] \rightarrow C$ with endpoints $a$ and $b$, that minimizes the following energy functional:

$$
E_{\text {principal }}(\vec{c})=\int_{0}^{1}\left|\frac{\vec{c}^{\prime}}{\sqrt{\left\langle\vec{c}^{\prime}, \vec{c}^{\prime}\right\rangle_{M}}}-\vec{V} \circ \vec{c}\right|_{M}^{2} d t=\int_{0}^{1}\left|\frac{\vec{\gamma}^{\prime}}{\left|\vec{\gamma}^{\prime}\right|}-\vec{G}(\vec{\gamma})\right|^{2} d t
$$


where $\vec{\gamma}=\vec{c} \circ \phi^{-1}:[0,1] \rightarrow D$ is the corresponding iteratively defined curve on the parameter domain; $\vec{G}(\vec{\gamma})=\sqrt{\lambda(\vec{\gamma})} \vec{V}(\vec{\gamma}) ;|\cdot|_{M}^{2}=<\cdot, \cdot>_{M}$ and $|\cdot|$ is the (usual) length defined on D. By minimizing the energy $E$, we minimize the difference between the tangent vector field along the curve and the principal direction field $\vec{V}$. The resulting minimizing curve is the curve that is closest to the curve traced along the principal direction. We can locate the landmark curves iteratively using the steepest descent algorithm.

\subsection{Landmark Hypothesis by Chan-Vese Segmentation}

In order to speed up the iterative scheme, we decided to obtain a good initialization by extracting the high curvature regions on the cortical surface using the Chan-Vese (CV) segmentation method [114]. We can extend the CV segmentation on $\mathbb{R}^{2}$ to any arbitrary Riemann surface $M$ such as the cortical surface.

We propose to minimize the following energy functional:

$$
F\left(c_{1}, c_{2}, \psi\right)=\int_{M}\left(u_{0}-c_{1}\right)^{2} H(\psi) d S+\int_{M}\left(u_{0}-c_{2}\right)^{2}(1-H(\psi)) d S+\nu \int_{M}\left|\nabla_{M} H(\psi)\right|_{M} d S,
$$

where $\psi: M \rightarrow \mathbb{R}$ is the level set function and $|\cdot|_{M}=\sqrt{<\cdot, \cdot>}$. The Euler-Lagrange equation becomes:

$$
\frac{\partial \zeta}{\partial t}=\lambda \delta(\zeta)\left[\nu \frac{1}{\lambda} \nabla \cdot\left(\sqrt{\lambda} \frac{\nabla \zeta}{\|\nabla \zeta\|}\right)-\left(w_{0}-c_{1}\right)^{2}+\left(w_{0}-c_{2}\right)^{2}\right]
$$

Now, the sulcal landmarks on the cortical surface lie at locations with relatively high curvature. To formulate the CV segmentation, we can consider the intensity term as being defined by the mean curvature. Sulcal locations can then be circumscribed by first extracting out the high curvature regions. Fixing two anchor points inside the extracted region, we can get a good initialization of the landmark curve by looking for a shortest path inside the region that joins the
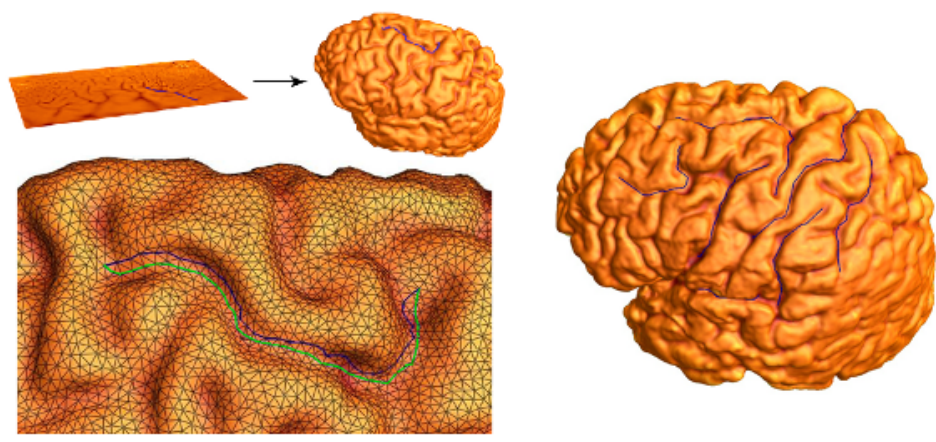

Fig. 3. Automatic landmark tracking using a variational approach. Left : we trace the landmark curves on the parameter domain along the edges whose directions are closest to the principal direction field, which gives a good initial guess of the landmark curve. Landmarks curve is then evolved to a deeper region using our variational approach. Right : Ten sulci landmarks are automatically traced using our algorithm. 
two points. Also, we can consider the umbilic points inside the region as anchor points. By definition, an umbilic point on a manifold is a location where the two principal curvatures are the same. Therefore, we can fix the anchor points inside the region by extracting regions with a small difference in principal curvatures.

\section{Optimization of Brain Conformal Parametrization}

In brain mapping research, cortical surface data are often mapped conformally to a parameter domain such as a sphere, providing a common coordinate system for data integration [51516]. As an application of our automatic landmark tracking algorithm, we use the automatically labelled landmark curves to generate an optimized conformal mapping on the surface, in the sense that homologous features across subjects are caused to lie at the same parameter locations in a conformal grid. This matching of cortical patterns improves the alignment of data across subjects. This is done by minimizing the compound energy functional $E_{\text {new }}=E_{\text {harmonic }}+\lambda E_{\text {landmark }}$, where $E_{\text {harmonic }}$ is the harmonic energy of the parameterization and $E_{\text {landmark }}$ is the landmark mismatch energy. Here, automatically traced landmark (continuous) curves are used and the correspondence are obtained using the unit speed reparametrization.

Suppose $C_{1}$ and $C_{2}$ are two cortical surfaces we want to compare. We let $f_{1}: C_{1} \rightarrow S^{2}$ be the conformal parameterization of $C_{1}$ mapping it onto $S^{2}$. Let $\left\{p_{i}:[0,1] \rightarrow S^{2}\right\}$ and $\left\{q_{i}:[0,1] \rightarrow S^{2}\right\}$ be the automatic labelled landmark curves, represented on the parameter domain $S^{2}$ with unit speed parametrization, for $C_{1}$ and $C_{2}$ respectively. Let $h: C_{2} \rightarrow S^{2}$ be any homeomorphism from $C_{2}$ onto $S^{2}$. We define the landmark mismatch energy of $h$ as: $E_{\text {landmark }}(h)=$ $1 / 2 \sum_{i=1}^{n} \int_{0}^{1}\left\|h\left(q_{i}(t)\right)-f_{1}\left(p_{i}(t)\right)\right\|^{2} d t$, where the norm represents distance on the sphere. By minimizing this energy functional, the Euclidean distance between the corresponding landmarks on the sphere is minimized.

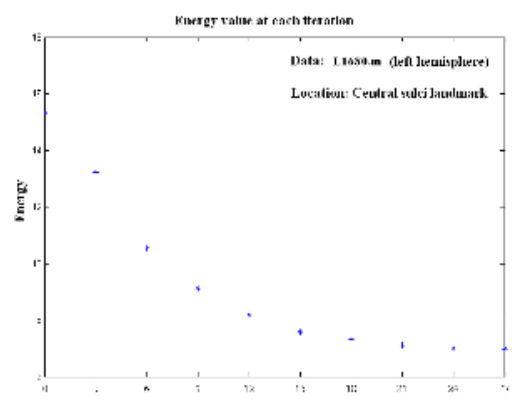

Difference between the two landmarks is measured by:
\[ E_{\text {difserence }}=\int_{0}^{1}\left\|\vec{c}_{\text {principal }}(t)-\vec{c}_{\text {manual }}(t)\right\|^{2} d t \]
\begin{tabular}{|l|l|l|l|}
\hline L1627.m & Central & Pre-central & Post-central \\
\hline Iteration 0 & 1.28 & 1.31 & 1.27 \\
\hline Iteration 30 & $0.21 \downarrow 83.6 \%$ & $0.22 \downarrow 83.2 \%$ & $0.21 \downarrow 83.5 \%$ \\
\hline L1680.m & Central & Pre-central & Post-central \\
\hline Iteration 0 & 1.33 & 1.35 & 1.26 \\
\hline Iteration 30 & $0.24 \downarrow 82.0 \%$ & $0.28 \downarrow 79.3 \%$ & $0.26 \downarrow 79.4 \%$ \\
\hline
\end{tabular}

Fig. 4. Left : The value of $E_{\text {principal }}$ at each iteration is shown. Energy reached its steady state with 30 iterations, meaning that our algorithm is efficient using the CV model as the initialization. Right : Numerical comparison between automatic labelled landmarks and manually labelled landmarks by computing the Euclidean distance $E_{\text {difference }}$ (on the parameter domain) between the automatically and manually labelled landmark curves. 

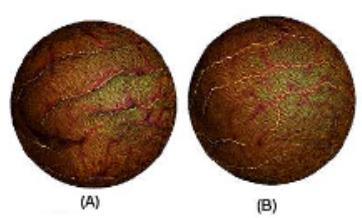

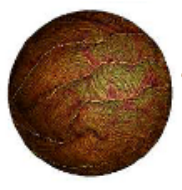

(C)

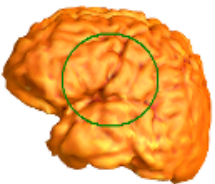

(D)

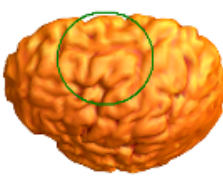

(E)

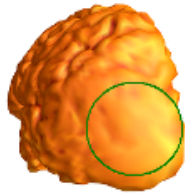

[F)

Fig. 5. Optimization of brain conformal mapping using automatic landmark tracking. In (A) and (B), two different cortical surfaces are mapped conformally to the sphere. In (C), we map one of the cortical surface to the sphere using our algorithm. (D), (E), (F) shows the average map of the optimized conformal parametrization using the variational approach with the automatically traced landmark curves. Observed that the important sulci landmarks are clearly shown.

\section{Experimental Results}

In one experiment, we tested our automatic landmark tracking algorithm on a set of left hemisphere cortical surfaces extracted from brain MRI scans, acquired from normal subjects at $1.5 \mathrm{~T}$ (on a GE Signa scanner). In our experiments, 6 landmarks were automatically located on cortical surfaces.

Figure 2(left), shows how we can effectively locate the initial landmark guess areas on the cortical surface using the Chan-Vese segmentation. Notice that the contour evolved to the deep sulcal region. In Figure 2(right), we locate the umbilic points in each sulcal region, which are chosen as the anchor points.

Our variational method to locate landmark curves is illustrated in Figure 3. With the initial guess given by the Chan-Vese model (we choose the two extreme points in the located area as the anchor points), we trace the landmark curves iteratively based on the principal direction field. In Figure 3 (left), we trace the landmark curves on the parameter domain along the edges whose directions are closest to the principal direction field. The corresponding landmark curves on the cortical surface is shown. Figure 3 (left) shows how the curve evolves to a deeper sulcal region with our iterative scheme. In Figure 3 (right), ten sulci landmarks are located using our algorithm. Our algorithm is quite efficient with the good intial guess using the CV-model. (See Fig 4 left)

To compare our automatic landmark tracing results with the manually labeled landmarks, we measured the Euclidean distance $E_{\text {difference }}$ (on the parameter domain) between the automatically and manually labelled landmark curves. Figure 4 (right) shows the value of $E_{\text {difference }}$ at different iterations for different landmark curves. Note that the value becomes smaller as the iterations proceed. This means that the automatically labeled landmark curves more closely resemble those defined manually as the iterations continue.

Figure 5 illustrates the application of our automatic landmark tracking algorithm. We illustrated our idea of the optimization of conformal mapping using the automatically traced landmark curves. Figure 5 (a) and (b) show two different cortical surfaces being mapped conformally to the sphere. Notice that the alignment of the sulci landmark curves are not consistent. In Figure 5 (c), the same cortical surface in (b) is mapped to the sphere using our method. Notice 
that the landmark curves closely resemble to those in (a), meaning that the alignment of the landmark curves are more consistent with our algorithm.

To visualize how well our algorithm can improve the alignment of the important sulci landmarks is, we took average of the 15 optimized conformal maps [14]. Figure 5 shows average maps at different angles. In (d) and (e), sulci landmarks are clearly preserved inside the green circle where landmarks are manually labelled. In (f), the sulci landmarks are averaged out inside the green circle where no landmarks are automatically detected. It means that our algorithm can help improving the alignment of the anatomical features.

\section{Conclusion and Future Work}

In this paper, we propose a variational method to automatically trace landmark curves on cortical surfaces, based on the principal directions. To accelerate the iterative scheme, we initialize the curves by extracting high curvature regions using Chan-Vese segmentation. This involves solving a PDE on the cortical manifold. The landmark curves detected by our algorithm closely resembled those labeled manually. Finally, we use the automatically labeled landmark curves to create an optimized brain conformal mapping, which matches important anatomical features across subjects. Surface averages from multiple subjects show that our computed maps can consistently align key anatomic landmarks. In future, we will perform a more exhaustive quantitative analysis of our algorithm's performance, mapping errors and quantifying improved registration, across multiple subjects, on a regional basis.

\section{References}

1. Vese, L., Chan, T. International Journal of Computer Vision 50 (2002) 271-293

2. Tao, X., Prince, J., Davatzikos, C. IEEE TMI 21 (2002) 513-524

3. Lohmann, G., Kruggel, F., von Cramon, D. IPMI 1230 (1997) 368-374

4. Zeng, X., Staib, L., Schultz, R., Win, L., Duncan, J. 3rd MICCAI (1999)

5. Gu, X., Wang, Y., Yau, S.T. IEEE TMI 23 (2004) 949-958

6. Glaunès, J., Vaillant, M., Miller, M. J. Maths. Imaging and Vision 20 (2004) $179-200$

7. Leow, A., Yu, C., Lee, S., Huang, S., Protas, H., Nicolson, R., Hayashi, K., Toga, A., Thompson, P. NeuroImage 24 (2005) 910-927

8. Thompson, P., Woods, R., Mega, M., Toga, A. Human Brain Mapping 9 (2000) 81-92

9. Schoen, R., Yau, S. International Press (1997)

10. Cipolla, R., Giblin, P.J. Cambridge University Press (2000)

11. Gu, X., Yau, S. ACM Symp on Geom. Processing 2003 (2003)

12. Wang, Y., Gu, X., Hayashi, K.M., Chan, T.F., Thompson, P.M., Yau, S.T. In: MICCAI. Volume II., Palm Springs, CA, USA (2005) 26-29

13. Rusinkiewicz, S. Symp. on 3D Data Processing, Vis., and Trans. (2004)

14. Lui, L., Wang, Y., Chan, T.F. VLSM, ICCV (2005)

15. Drury, H., Essen, D.V., Corbetta, M., Snyder, A. In:. Academic Press (1999)

16. Fischl, B., Sereno, M., Tootell, R., Dale, A. In: Human Brain Mapping. Volume 8. (1999) 272-284 